\title{
Radiographic markers of clinical outcomes after endoscopic third ventriculostomy with choroid plexus cauterization: cerebrospinal fluid turbulence and choroid plexus visualization
}

\author{
Jonathan Pindrik, MD, ${ }^{1}$ Brandon G. Rocque, MD, MS, ${ }^{2}$ Anastasia A. Arynchyna, MPH, ${ }^{2}$ \\ James M. Johnston, MD, ${ }^{2}$ and Curtis J. Rozzelle, MD² \\ 'Division of Pediatric Neurosurgery, Nationwide Children's Hospital and The Ohio State University Wexner Medical Center, \\ Columbus, Ohio; and 'Division of Pediatric Neurosurgery, Children's of Alabama and University of Alabama at Birmingham, \\ Alabama
}

OBJECTIVE Endoscopic third ventriculostomy (ETV) with choroid plexus (CP) cauterization (CPC) represents a viable treatment option for congenital hydrocephalus in infants younger than 2 years. Imaging studies complement clinical data in the evaluation of treatment success or failure. The objectives of this study were to investigate novel radiographic markers-cerebrospinal fluid (CSF) turbulence and CP visualization-and their ability to reflect or predict clinical outcomes following ETVICPC.

METHODS Hydrocephalic patients younger than 2 years who were initially treated by ETVICPC at the senior authors' institution between March 2013 and February 2014 were retrospectively reviewed. Clinical data, as well as the visualization of CSF turbulence and CP on pre- and postoperative fast-sequence MRI, were recorded. Radiographic images were reviewed by a blinded observer based on specific criteria for the visualization of CSF turbulence and CP. Data were collected and analyzed using descriptive statistics, including Fisher's exact test for comparisons. The research team obtained appropriate institutional review board approval for this study, without the need for informed consent.

RESULTS Among the 32 patients (53\% male and $47 \%$ female) studied, 18 of $32(56 \%)$ responded favorably to initial or repeat ETVICPC, with 13 of 32 (41\%) patients requiring 1 surgery. Of the $19(59 \%)$ patients whose initial ETV/CPC failed, 8 of $19(42 \%)$ patients underwent repeat ETVICPC, with 5 of 8 (63\%) patients responding favorably. Radiographic CSF turbulence appeared more frequently following ETVICPC failure than after ETVICPC success $(55 \%$ vs $18 \%$, respectively; $p=0.02$ ). The sensitivity and specificity of CSF turbulence as a radiographic marker for ETV/CPC failure were $80 \%$ and $58 \%$, respectively. The radiographic depiction of CP disappearance following ETVICPC from pre- to postoperative imaging occurred in 20 of 30 patients (67\%). Among the patients who responded unsuccessfully to ETV/CPC and ultimately required secondary shunt insertion, $71 \%$ (10 of 14 patients) demonstrated CP persistence on postoperative imaging. In contrast, $6 \%$ (1 of 18) of patients who were treated successfully by ETVICPC demonstrated the presence of CP on follow-up imaging. This difference reached statistical significance $(p=0.0001)$. The visualization of CP persistence despite ETVICPC reflected treatment failure with $91 \%$ sensitivity and $81 \%$ specificity. The sensitivity of either or both radiographic markers to suggest ETVICPC failure was $77 \%$, while their specificity (both markers absent, thereby indicating ETV/CPC success) was $81 \%$.

CONCLUSIONS Radiographic markers correlate with clinical outcomes following the treatment of infantile hydrocephalus with ETVICPC. Specifically, CSF turbulence may indicate ongoing pathological CSF flow dynamics, while CP absence following ETV/CPC may predict shunt independence. Future studies that incorporate prospective review and formal intra- and interobserver reliability estimates may help corroborate the utility of these radiographic markers.

http://thejns.org/doi/abs/10.3171/2016.3.PEDS15364

KEY WORDS CSF turbulence; choroid plexus; endoscopic third ventriculostomy; choroid plexus cauterization; radiographic markers; hydrocephalus

ABBREVIATIONS CP = choroid plexus; $\mathrm{CPC}=$ choroid plexus cauterization; $\mathrm{ETV}=$ endoscopic third ventriculostomy; FS-MRI = fast-sequence MRI; IVH = intraventricular hemorrhage; $\mathrm{MMC}=$ myelomeningocele.

SUBMITTED June 15, 2015. ACCEPTED March 14, 2016

INCLUDE WHEN CITING Published online May 13, 2016; DOI: 10.3171/2016.3.PEDS15364 
$\mathrm{W}$ ITH the addition of choroid plexus cauterization (CPC), endoscopic third ventriculostomy (ETV) has increased in popularity in recent years as an acceptable and potentially favorable treatment modality for hydrocephalus in young children. The application of ETV combined with CPC (ETV/CPC) to neonates or infants with new-onset hydrocephalus due to various etiologies represents a recent development both nationally and globally. Despite potentially lower success rates in this patient population, ETV/CPC provides an alternative treatment modality for CSF diversion that avoids the risks inherent to permanent indwelling shunt hardware.

Radiographic examinations contribute significantly to the evaluation of patients with previously treated hydrocephalus. The combination of imaging and clinical data may reveal inadequate or unsuccessful treatment and the need for surgical revision. Failure of initial ETV/CPC can be managed with repeat endoscopic exploration and ETV, along with more extensive CPC as necessary. Alternatively, secondary shunt insertion may be performed, with or without preceding endoscopic reexploration.

Various radiographic markers that correlate with ETV success or failure have been used clinically and explored in the recent literature. These radiographic findings include ventriculostomy-site "flow void" and third ventricular parameters, such as floor contours, width, and midsagittal cross-sectional area. ${ }^{6}$ These markers corroborate clinical findings in order to help determine if a patient has responded favorably to ETV/CPC or requires further treatment, either with repeat endoscopy or shunt implantation. This study investigates 2 additional imaging findings in the setting of ETV/CPC: CSF turbulence and choroid plexus $(\mathrm{CP})$ visualization. We explored if radiographic evidence of CSF turbulence and CP visualization correlated with clinical outcomes following ETV/CPC.

\section{Methods}

A retrospective review of MRI scans obtained previously for clinical purposes in hydrocephalic patients under 2 years of age was performed. The imaging modality mainly consisted of shunt function or fast-sequence MRI (FS-MRI) incorporating single-shot T2-weighted turbo spin-echo sequences (axial, coronal, and sagittal). The study patients underwent initial treatment for hydrocephalus via ETV/CPC at the our institution from March 2013 through February 2014. The clinical records of these patients were reviewed to determine the initial operative dates, clinical outcomes, and dates of repeat surgery for hydrocephalus (repeat ETV or secondary shunt insertion), when applicable. Patients with preoperative standard MRI or FS-MRI, postoperative FS-MRI, and documented postoperative clinical follow-up evaluations were selected for this study. Because this study investigated both success and failure following neuroendoscopic treatment, a minimum duration of clinical follow-up was not incorporated as an exclusion criterion. The research team obtained appropriate institutional review board approval for this study, without the need for informed consent.

The imaging sequences were selected for review based on specific criteria regarding ETV/CPC outcome and tim- ing. FS-MRI scans were designated as reflecting ETV/ CPC failure if repeat surgery was performed within 2 weeks from the imaging date. For the investigation of CSF turbulence, the latest FS-MRI scan available for review (in cases of ETV/CPC success) and the last FS-MRI examination prior to repeat surgical intervention (when applicable, and while fulfilling the above criterion) were selected. In this fashion, a study patient who responded favorably to repeat neuroendoscopic treatment may have had up to 2 imaging examinations included (1 "failed" FS-MRI scan preceding repeat surgery and 1 "successful" FS-MRI scan that occurred at the latest follow-up). Similarly, a study patient undergoing repeat ETV/CPC followed by shunt insertion may offer 2 FS-MRI scans for review (1 "failed" scan preceding repeat neuroendoscopy and 1 "failed" scan preceding shunt insertion). Patients managed with secondary shunt insertion following initial ETV/CPC failure would contribute 1 imaging examination for review (1 "failed" scan preceding shunt insertion), as would patients who responded favorably to the initial ETV/CPC (1 "successful" scan at the latest follow-up). This mode of image selection led to a data analysis for CSF turbulence recognition on a per-patient or per-scan basis, depending on ETV/CPC outcome.

For the evaluation of $\mathrm{CP}$ visualization, the latest followup FS-MRI scan available for review was selected, with a minimum requirement of image acquisition being performed at least 4 weeks from the initial ETV/CPC date. In contrast to image selection for CSF turbulence, this selection process provided 1 FS-MRI scan per study patient for review, which was stratified by clinical ETV/CPC outcome (ETV/CPC "success" reflecting shunt independence, or ETV/CPC "failure" indicating eventual shunt insertion). The minimum 4-week requirement was incorporated to allow sufficient time for the disappearance or involution of the $\mathrm{CP}$ glomus following CPC. Without this constraint, imaging in the setting of early ETV/CPC failure might create a false correlation between treatment failure and residual CP that has not yet become radiographically occult. The latest follow-up FS-MRI scan seemed the most reasonable selection for review since it provided the greatest time interval to observe the effects of CPC, provided a consistent method of image selection, and because $\mathrm{CP}$ visualization should not be expected to change from "absent" to "present" over the serial scans that preceded the latest imaging study. The serial FS-MRI scans that occurred prior to the last follow-up examination were reviewed to check for internal consistency with respect to $\mathrm{CP}$ visualization (CP "presence" on the last follow-up scan should be matched by CP "presence" on prior scans, while $\mathrm{CP}$ "absence" should not be followed by CP "presence" on subsequent scans).

The surgical approach remained consistent among the pediatric neurosurgeons on the study team. During the initial treatment of hydrocephalus, standard ETV was performed via a coronal entry site (typically on the right side) on the midpupillary line. This site often coincided with the lateral apex of the anterior fontanelle, thereby obviating bone removal; however, removal of adjacent or surrounding bone was performed with rongeurs or a highspeed drill as necessary. Following linear dural opening 
(when possible), the flexible endoscope was advanced into the lateral ventricle and through the foramen of Monro after identifying intraventricular anatomy. Ventriculostomy was performed through the floor of the third ventricle, anterior to the impression of the mammillary bodies, and posterior to the infundibular recess and the impression of the dorsum sellae. Following ETV and exploration of the prepontine cistern with lysis of the membrane of Liliequist and any arachnoid adhesions, extensive CPC was performed in the ipsilateral and contralateral bodies, atria, and temporal horns of both lateral ventricles. Additional time and focus was devoted to thorough coagulation of the $\mathrm{CP}$ glomus bilaterally. Following thorough CPC, the ventriculostomy site was reexplored for patency. The durotomy was closed primarily (when possible), and the wound was closed in a standard 2-layer fashion.

For recurrent or progressive hydrocephalus, the attending surgeons would evaluate the necessity for repeat treatment based on the clinical (symptoms of elevated intracranial pressure, accelerated head growth, fullness or bulging of the anterior fontanel, suture diastasis) and imaging information. Similarly, the attending surgeons decided to perform repeat endoscopic treatment versus secondary shunting at their discretion. Typically, the patient would be positioned to allow for a repeat endoscopic exploration first and ventricular shunt insertion as necessary. The attending surgeon additionally assessed the need for repeat or expanded CPC (typically not required) following repeat ETV.

FS-MRI scans were reviewed by 1 blinded observer for the presence or absence of 2 markers: CSF turbulence and $\mathrm{CP}$ visualization. For this pilot study, the single observer specialized in pediatric neurosurgery at the fellowship level and is a contributing author (J.P.). Visualization of CSF turbulence entailed 3 requirements (Fig. 1): 1) the presence of an irregular, heterogeneous, or hypointense signal intensity in a pattern that suggested nonlinear CSF flow; 2) the presence of this signal intensity in at least 2 of 3 supratentorial ventricles; and 3) the distinction of this signal intensity from imaging artifact due to patient movement. Similarly, radiographic evaluation of CP entailed specific designations (Fig. 2): 1) the clear visualization of the glomus in at least 1 ventricular atrium denoting $\mathrm{CP}$ "presence"; and 2) the lack of or diminutive size of the glomus in both ventricular atria denoting CP "absence." These radiographic criteria were established prior to imaging review.

The qualitative determinants of CSF turbulence and $\mathrm{CP}$ visualization were used based on the capabilities and limitations of FS-MRI, which is the preferred imaging study for neonates and infants who require radiographic evaluation following treatment for CSF diversion at the senior author's (C.J.R.) institution. Subjective interpretation of the lack of a clearly visualized CP glomus, or only scant CP glomus remaining within the ventricular atria, served as surrogate markers for $\mathrm{CP}$ absence. The observer was blinded to both patient outcome and the chronological order of the MRI scans (i.e., at which point in the patient's clinical course the radiographic examination occurred). Primary outcome variables included ETV/CPC clinical outcome and radiographic visualization of CSF turbulence and $\mathrm{CP}$.

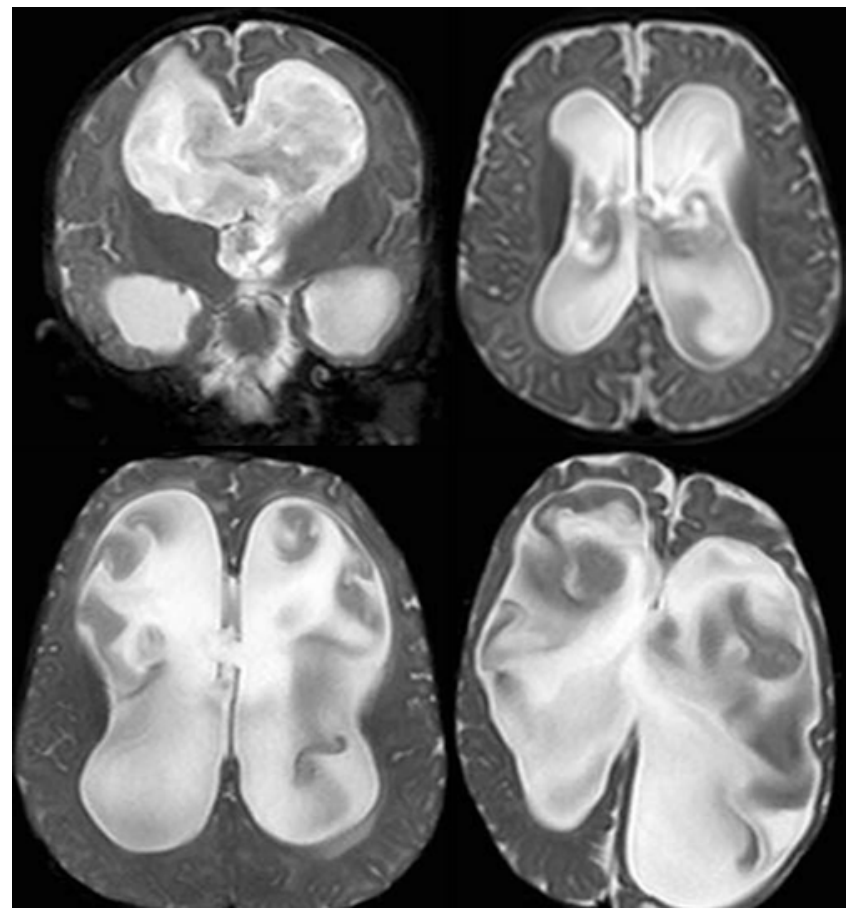

FIG. 1. Examples of radiographic CSF turbulence. These coronal and axial T2-weighted FS-MRI slices demonstrate the presence of radiographic CSF turbulence in at least 2 of 3 supratentorial ventricles.

Data were collected and analyzed using descriptive statistics, including Fisher's exact test for comparisons. The sensitivity and specificity of CSF turbulence and CP visualization as radiographic markers for ETV/CPC failure were calculated as well. Serial imaging examinations that were evaluated for $\mathrm{CP}$ visualization were reviewed for internal consistency and as a surrogate method for testing intraobserver reliability. Specifically, the number of imaging examinations following any postoperative FS-MRI scan with CP "absence" was tabulated for all patients. Of these scans, the number of imaging studies demonstrating an inconsistent result (CP "presence" following a previous designation of CP "absence") was identified. The number of inconsistent results was compared with the total number of scans to estimate a crude measure of internal consistency.

\section{Results}

This study incorporated 32 patients (53\% male and $47 \%$ female) with a mean patient age at first ETV/CPC of 17.1 weeks (SD 20.0 weeks; range 0.4-96.9 weeks) (representing the actual age since birth). The etiologies of hydrocephalus included prematurity with intraventricular hemorrhage (IVH) (47\%), myelomeningocele (MMC) (19\%), congenital aqueductal stenosis (19\%), and other causes (compressive arachnoid cyst, obstructive hydrocephalus due to tumor, meningitis, and congenital communicating hydrocephalus) (16\%) (Table 1). With a mean follow-up of 10.6 months (SD 4.3 months; range 1.0-21.0 months), approximately $56 \%$ (18 of 32) of patients responded favorably to ETV/CPC (initial or repeat surgery), thereby 


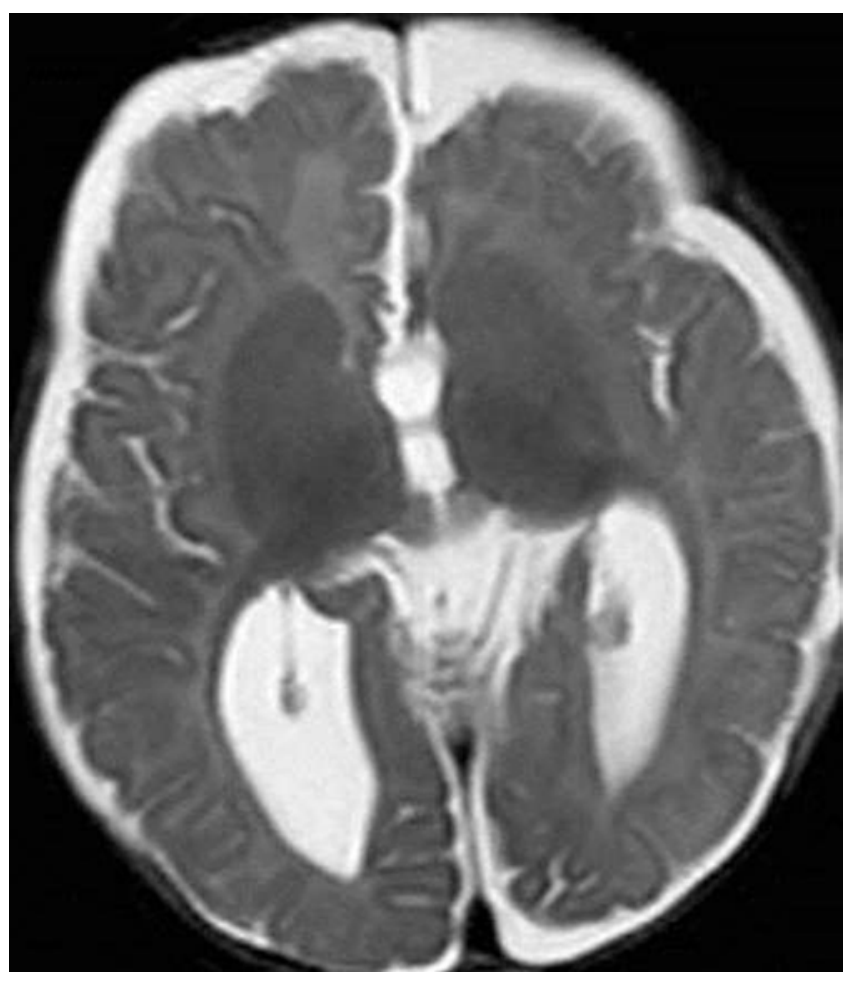

FIG. 2. Example of radiographic CP presence. This axial T2-weighted FS-MRI slice demonstrates the typical appearance of the CP glomus within at least 1 ventricular atrium, which is consistent with the study's definition of CP "presence."

remaining shunt independent (Fig. 3). Patients with MMC (5 of 6 patients; 83\%) and congenital aqueductal stenosis ( 5 of 6 patients; $83 \%$ ) exhibited the highest ETV/CPC success rates, while premature neonates with IVH (6 of 15 patients; 40\%) demonstrated the lowest ETV/CPC success rate (Fig. 3). Initial ETV/CPC carried a 41\% (13 of 32 patients) success rate, while $63 \%$ (5 of 8 ) of patients who underwent repeat ETV/CPC remained shunt independent (Fig. 3). Following initial ETV/CPC failure, 58\% (11 of 19) of patients underwent secondary shunt insertion (Fig. 3). Repeat surgical intervention occurred at a mean duration of 7.8 weeks (range 1.1-26.6 weeks) following initial ETV/ CPC failure and 2.2 weeks (range 2.0-2.4 weeks) following unsuccessful repeat neuroendoscopy. CSF turbulence appeared in 55\% (12 of 22) of imaging examinations performed in study patients who experienced ETV/CPC failure, while appearing in 18\% (3 of 17) of FS-MRI scans from study patients who responded favorably to ETV/CPC (Table 2; Fig. 4-5). This difference reached statistical significance ( $\mathrm{p}=0.02$; Fisher's exact test). The sensitivity of CSF turbulence as a radiographic marker for ETV/CPC failure was $80 \%$, while the specificity was $58 \%$ (Table 2). The radiographic suggestion of CSF turbulence appeared in 1 study patient's pretreatment MRI ( 1 of 32 patients; $3 \%$ ). The mean duration of time between initial ETV/CPC and imaging prior to repeat surgery was 7.5 weeks (range 0.6-26.4 weeks), while the average time interval between repeat neuroendoscopy and subsequent imaging prior to VP shunt insertion (which only occurred in 3 patients)
TABLE 1. Sex of the study population and etiology of hydrocephalus

\begin{tabular}{lc}
\hline \multicolumn{1}{c}{ Variable } & No. of Patients (\%) \\
\hline Sex & \\
\hline Male & $17(53)$ \\
\hline Female & $15(47)$ \\
\hline Etiology & $15(47)$ \\
\hline Prematurity w/ IVH & $6(19)$ \\
\hline MMC & $6(19)$ \\
\hline Congenital aqueductal stenosis & $5(16)$ \\
\hline Other*
\end{tabular}

* Includes arachnoid cyst, tumor, and meningitis.

was 1.9 weeks (range 1.9-2.0 weeks). The latest follow-up imaging in the cases of ETV/CPC success (with initial or repeat surgery) occurred on average at 30.0 weeks (range 4.9-62.7 weeks) following the most recent neuroendoscopic procedure.

The radiographic depiction of adequate CPC (CP "presence" changing to CP "absence") occurred after 67\% (20 of 30) of neuroendoscopic procedures (incorporating those with the appropriate pre- and postoperative imaging studies) (Fig. 6). Residual CP appeared in $71 \%$ (10 of 14) of the latest follow-up FS-MRI scans in patients whose ETV/CPC failed and ultimately required shunt insertion (Table 3 ). In contrast, CP was present in 6\% (1 of 18) of the latest follow-up FS-MRI scans in patients who responded favorably to ETV/CPC and remained shunt independent. This difference reached statistical significance $(\mathrm{p}=$ 0.0001; Fisher's exact test) (Table 3). The sensitivity of CP visualization as a radiographic marker of ETV/CPC failure reached $91 \%$, while the specificity was $81 \%$ (Table 3). The mean duration of time between the initial ETV/CPC and latest follow-up imaging for $\mathrm{CP}$ visualization evaluation was 23.0 weeks (range 4.0-62.7 weeks).

Seven study patients $(22 \%)$ underwent repeat surgical intervention prior to the 4-week minimum time constraint imposed on radiographic CP evaluation. Within this subset of patients, the average duration of time between the initial and repeat surgery was 2.1 weeks (range 1.1-3.7 weeks). Following the study protocol, the latest followup FS-MRI scan was still used for CP evaluation in these patients, and the preceding imaging examinations were checked for internal consistency. One patient demonstrated CP "absence" at 1.4 weeks following surgery and at the latest follow-up scan (4.4 weeks). Five study patients consistently exhibited CP persistence on all postoperative scans, including the early and latest follow-up imaging examinations. Within this subset of 7 patients, 1 patient demonstrated an inconsistent finding of CP "absence" on the early postoperative scan (3.6 weeks from surgery), while exhibiting CP "presence" on all subsequent imaging studies (including the latest follow-up scan at 9.6 weeks after the initial ETV/CPC). One additional study patient (outside of this subset) demonstrated a similar inconsistent finding on the postoperative FS-MRI scans. Based on the method described above, this resulted in an internal incon- 


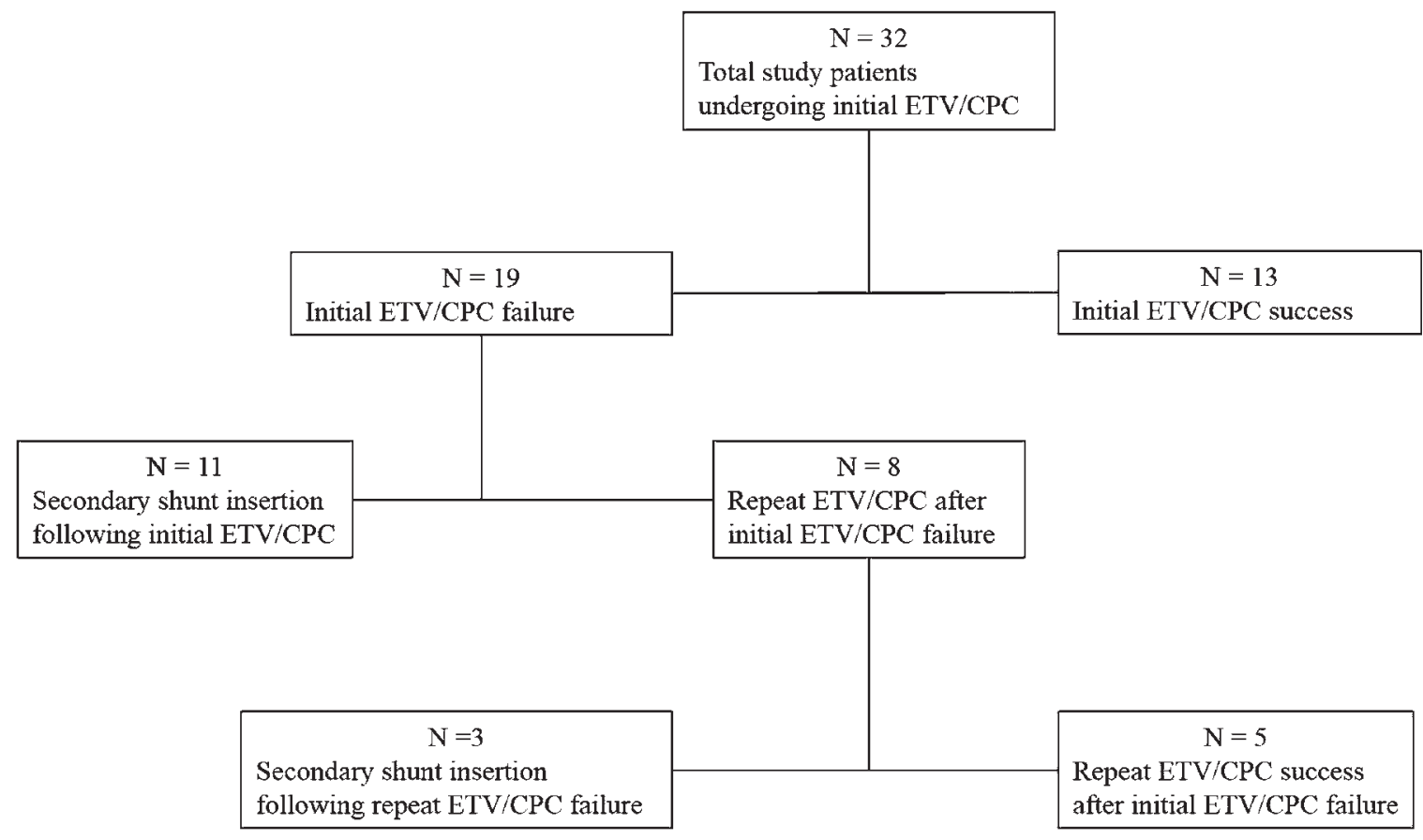

FIG. 3. Flow diagram for this study's patient cohort. This flow diagram demonstrates the total study patient cohort stratified by ETVICPC success or failure (on initial or repeat attempts) and the performance of secondary shunt insertion (when necessary).

sistency rate of approximately 9\% (2 inconsistent scans of 22 total postoperative scans following CP disappearance). This measure of $91 \%$ internal consistency may be considered a crude but surrogate marker of intraobserver reliability for the specific type of FS-MRI evaluation described here.

Regarding the visualization of the radiographic markers in combination, the presence of both CSF turbulence and $\mathrm{CP}$ (following the same criteria for the evaluation of each marker individually) always reflected ETV/CPC failure (4 of 4 postoperative scans), but this combination occurred in only 4 of 20 (20\%) postoperative "failed" FSMRI scans. The presence of either or both markers occurred in 17 of 20 (85\%) imaging studies of patients demonstrating ETV/CPC failure, while occurring in 5 of 18 (28\%) scans of patients who responded favorably to ETV/ CPC (Table 4). This difference reached statistical significance $(\mathrm{p}=0.0007$; Fisher's exact test). The sensitivity and

TABLE 2. CSF turbulence as a radiographic marker of clinical outcomes following ETV/CPC*

\begin{tabular}{cccccc}
\hline & & ETVI & & & \\
Study Cohort & $\begin{array}{c}\text { ETVICPC } \\
\text { Success }\end{array}$ & $\begin{array}{c}\text { CPC } \\
\text { Failure }\end{array}$ & $\begin{array}{c}p \\
\text { Value }\end{array}$ & Sensitivity & Specificity \\
\hline $\begin{array}{c}\text { CSF turbulence } \\
\text { present }\end{array}$ & $3(18)$ & $12(55)$ & 0.02 & $12(80)$ & NA \\
\hline $\begin{array}{c}\text { CSF turbulence } \\
\text { absent }\end{array}$ & $14(82)$ & $10(45)$ & NA & NA & $14(58)$ \\
\hline
\end{tabular}

$\mathrm{NA}=$ not applicable.

* Values are reported as the number of patients (\%) unless stated otherwise. specificity of using either or both radiographic markers as an indication of ETV/CPC failure were $77 \%$ (17 of 22) and $81 \%$ (13 of 16), respectively (Table 4).

\section{Discussion}

Radiographic markers can be used to help reflect or predict the clinical outcomes of pediatric patients with hydrocephalus following ETV or combined ETV/CPC. These represent important adjuncts for evaluation and management in the neonatal population due to the occasional paucity of clinical markers of success or failure.
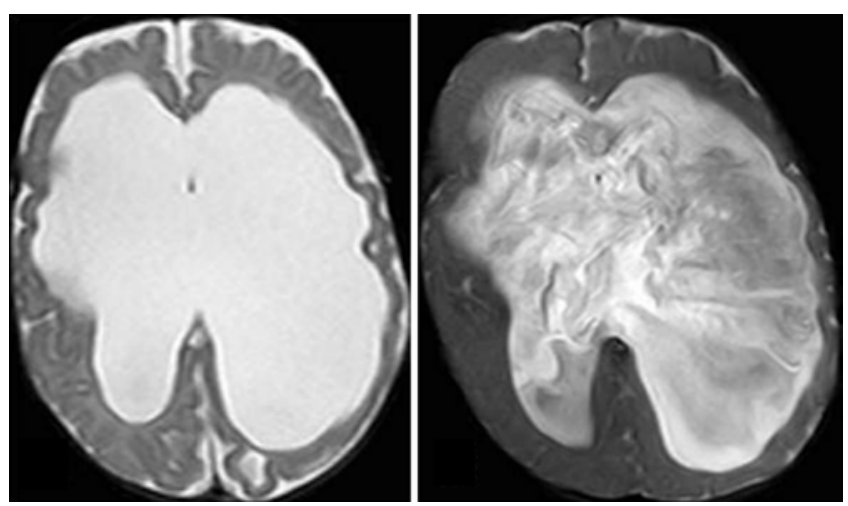

FIG. 4. Presence of radiographic CSF turbulence in the setting of treatment failure. Left: This axial T2-weighted FS-MRI slice acquired during the period of initial success following ETVICPC lacks any radiographic signs of CSF turbulence. Right: However, follow-up imaging more than 5 months later (in the same study patient) in the setting of delayed ETVI $\mathrm{CPC}$ failure demonstrates findings consistent with CSF turbulence. 

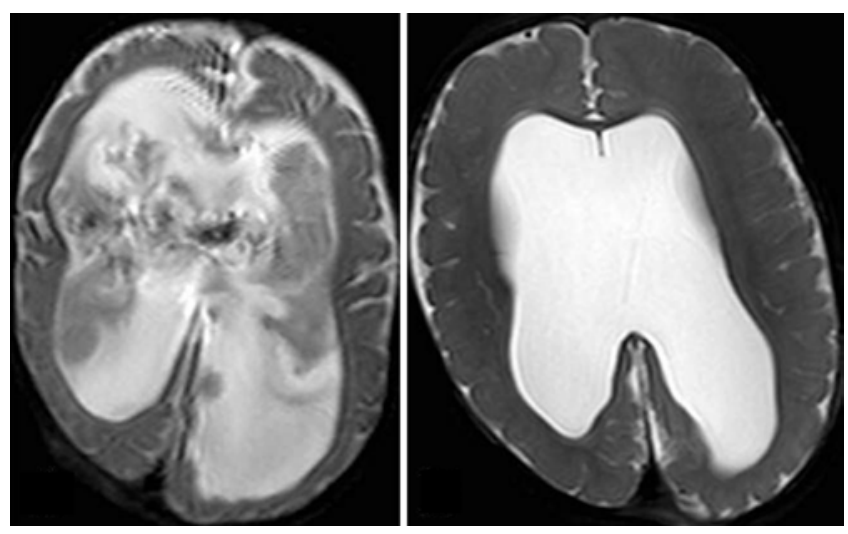

FIG. 5. Radiographic investigation of CSF turbulence in the setting of treatment failure and success. These axial T2-weighted FS-MRI slices demonstrate the presence of CSF turbulence in the setting of ETVICPC failure (left), with the resolution of CSF turbulence following clinically successful treatment by ventriculoperitoneal shunting (right) in the same study patient.

Previously studied radiographic characteristics of ETV site patency or ventricular size include a CSF flow void traversing the third ventriculostomy site, computerized ventricular volume analysis, the frontal-occipital horn ra-
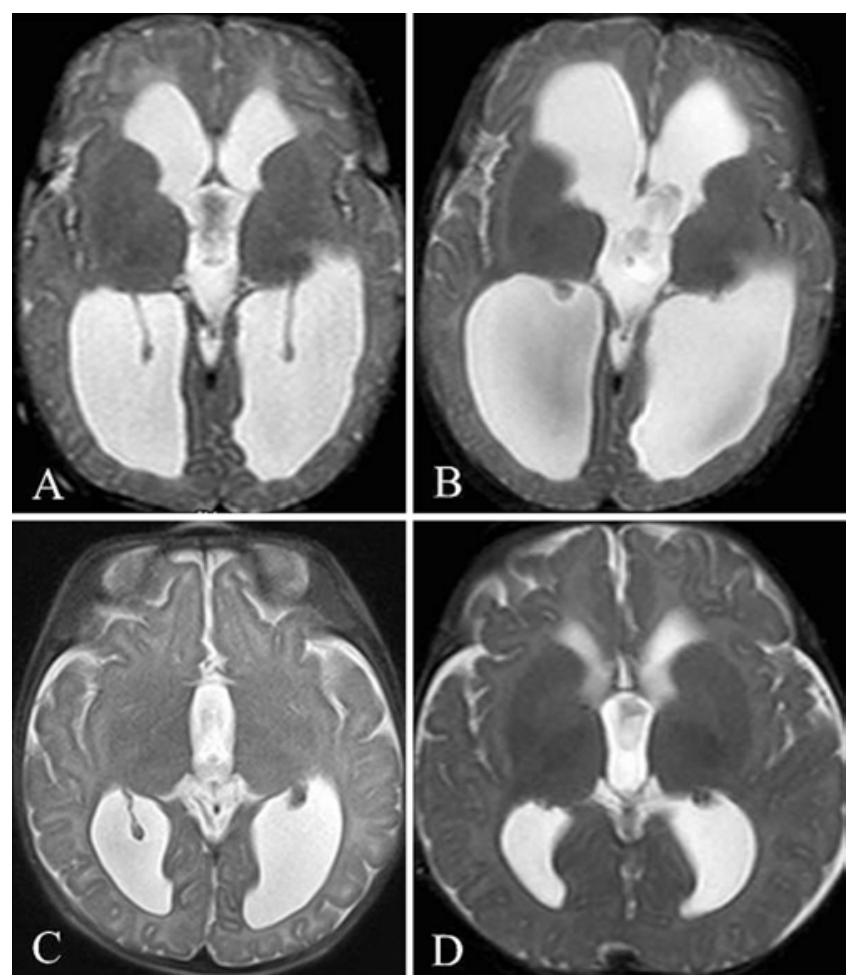

FIG. 6. Radiographic depiction of the CP before and after ETV/CPC. These axial T2-weighted FS-MRI slices reflect CP "presence" before (A and C) and CP "absence" (based on study definitions) after (B and D) clinically successful ETVICPC in the 2 respective study patients. The duration of time between serial imaging scans was approximately 12 weeks (A and B) and 9 weeks (C and D) in the same study patients, respectively.
TABLE 3. CP visualization as a radiographic marker of clinical outcomes following ETVICPC*

\begin{tabular}{cccccc}
\hline Study Cohort & $\begin{array}{c}\text { ETVICPC } \\
\text { Success }\end{array}$ & $\begin{array}{c}\text { ETVICPC } \\
\text { Failure }\end{array}$ & $\begin{array}{c}\mathrm{p} \\
\text { Value }\end{array}$ & Sensitivity & Specificity \\
\hline CP present & $1(6)$ & $10(71)$ & 0.0001 & $10(91)$ & NA \\
\hline CP absent & $17(94)$ & $4(29)$ & NA & NA & $17(81)$ \\
\hline * Values are reported as the number of patients (\%) unless stated otherwise.
\end{tabular}

tio, third ventricular width, and third ventricular midsagittal cross-sectional area. ${ }^{1,2,5,6}$ Despite the functional diversion of CSF flow when successful, ETV produces more subtle ventricular size changes than traditional shunting. ${ }^{6,11}$ This study presents 2 additional radiographic markersCSF turbulence and CP visualization - that may be used to reflect clinical outcomes in hydrocephalic neonates and infants following ETV/CPC. These radiographic markers have not been reported or previously studied in children treated for hydrocephalus.

\section{CSF Turbulence}

The radiographic appearance of turbulent CSF flow represents an imaging artifact, which is typically best appreciated on T2-weighted MRI sequences (including the FS-MRI turbo spin-echo sequences used in this study) given the relative hyperintensity of CSF (Figs. 1, 4, and 5). ${ }^{9,10}$ Signal loss due to elevated CSF flow velocity or turbulence has been observed clinically and reported in both humans and dogs. ${ }^{7,8}$ For instance, Sherman and Citrin reported the frequent occurrence of CSF flow voids or signal dropout within the cerebral aqueduct of Sylvius and the fourth ventricle in MRI studies of adult patients without intraventricular abnormalities. ${ }^{8}$ The cerebral aqueduct represents a common location for CSF flow voids due to the increased velocity of the flow from a larger volume (third ventricle) through a region with a comparatively smaller cross-sectional area (cerebral aqueduct). ${ }^{9,10}$ The pulsatile flow-related parameters of high velocity and turbulence produce alterations in CSF signal intensity by various mechanisms. The radiographic appearance of CSF turbulence reflects motion-induced phase changes during MR image acquisition, with explanations extending beyond the scope of this article. In simplistic terms, turbulence involves multiple, randomly oriented velocity vectors of CSF flow with different signal phases, resulting in a het-

TABLE 4. Combination of CSF turbulence and CP visualization as radiographic markers for clinical outcomes following ETVICPC*

\begin{tabular}{|c|c|c|c|c|c|}
\hline Study Cohort & $\begin{array}{c}\text { ETVICPC } \\
\text { Success }\end{array}$ & $\begin{array}{l}\text { ETV/CPC } \\
\text { Failure }\end{array}$ & $\begin{array}{c}p \\
\text { Value }\end{array}$ & Sensitivity & Specificity \\
\hline $\begin{array}{c}\text { Either or both } \\
\text { markers } \\
\text { present }\end{array}$ & $5(28)$ & $17(85)$ & 0.0007 & $17(77)$ & NA \\
\hline $\begin{array}{c}\text { Both markers } \\
\text { absent }\end{array}$ & $13(72)$ & $3(15)$ & NA & NA & $13(81)$ \\
\hline
\end{tabular}

* Values are reported as the number of patients (\%) unless stated otherwise. 
erogeneous pattern of signal loss. ${ }^{7,9,10}$ Consequently, turbulent nonlaminar flow produces CSF flow voids and signal heterogeneity. ${ }^{4,9}$

As demonstrated by the findings in this article, the radiographic appearance of CSF turbulence may reflect underlying pathological CSF flow dynamics and/or reduced intracranial compliance, signaling the potential failure of ETV/CPC in certain patients. ${ }^{7,10}$ Prior studies have reported higher incidences of CSF flow void signs and signal heterogeneity in patients with ventriculomegaly as compared with individuals with normal-sized ventricles. ${ }^{9,10}$ In this study, radiographic evidence of CSF turbulence appeared in $55 \%$ of patients following ETV/CPC failure, as compared with $18 \%$ of patients following successful endoscopic treatment, and this reached statistical significance ( $p=0.02$; Table 2). A CSF flow void through the ETV site typically suggests patency and provides reassurance. ${ }^{9,10}$ In contrast, the distinct radiographic appearance of CSF turbulence as described in this study may reflect pathological CSF flow dynamics and signal treatment failure with a modest sensitivity of $80 \%$ (Table 2).

The radiographic depiction of CSF turbulence might reflect the elevated amplitudes of the intraventricular CSF pulsations thought to be instrumental in progressive ventriculomegaly in infants with hydrocephalus. ${ }^{12,13}$ While ETV may function as a pulsation absorber, progressive stenosis or scarring at the ventriculostomy site reduces its pulsation-absorption capacity. ${ }^{13}$ Scarring across an opened but previously undisturbed anatomical surface (third ventricular floor) might sufficiently influence ventricular compliance to produce CSF pulsation artifacts such as turbulent flow. The new appearance of CSF turbulence in a hydrocephalic infant's FS-MRI following ETV/CPC could represent the progression of inadequately treated hydrocephalus. Cauterization of the CP might also function by reducing the amplitude of the intraventricular CSF pulsations, as previously described. ${ }^{12,13}$ Based on the hydrodynamic CSF pulsation model that was proposed previously in the literature, ${ }^{12,13}$ it follows that CSF turbulence-which reflects pathological CSF flow dynamics-and CP persistence despite ETV/CPC might represent useful radiographic markers of treatment failure.

\section{CP Visualization}

Visualization of the $\mathrm{CP}$ extends the utility of radiographic markers following ETV/CPC or when considering secondary shunt insertion (Figs. 2 and 6). Among the patients exhibiting clinical ETV/CPC failure, 71\% (10 of 14) demonstrated the persistence of $\mathrm{CP}$ on postoperative imaging, while only $6 \%$ (1 of 18 ) of patients who responded favorably to ETV/CPC demonstrated CP persistence, thereby representing a statistically significant difference $(p=0.0001$; Table 3). In this study, CP presence on postoperative FS-MRI reflected clinical ETV/CPC failure and predicted ultimate secondary shunt insertion with reasonably high sensitivity (91\%). Additionally, the absence of $\mathrm{CP}$ following ETV/CPC - suggesting radiographically adequate $\mathrm{CPC}$ - predicted ETV/CPC success and shunt independence with moderate specificity (81\%). While demonstrating the potential utility of $\mathrm{CP}$ visualization as a radiographic marker, this finding also emphasizes the importance of CPC in the treatment of hydrocephalic neonates, as suggested by prior works..$^{13,14}$

Of those individuals (10 of 30 patients; 33\%) with persistent CP following ETV/CPC, $70 \%$ (7 of 10 patients) had developed hydrocephalus due to prematurity with IVH. Six $(86 \%)$ of these 7 patients with prematurity, posthemorrhagic hydrocephalus and CP persistence despite ETV/ $\mathrm{CPC}$ ultimately required shunt insertion. The high rate of $\mathrm{CP}$ persistence in this cohort of IVH patients may reflect the difficulty of achieving extensive CPC due to scarring of the $\mathrm{CP}$ in this population. Furthermore, the relatively low success rate $(40 \%)$ of ETV/CPC and high shunting rate of neonates with prematurity and IVH in this small sample reflects the clinical experience of the study authors.

The radiographic demonstration of $\mathrm{CP}$ persistence or disappearance following ETV/CPC has not been previously reported or studied in the literature for comparison. However, near-complete CPC has been shown to correlate with higher rates of treatment success in hydrocephalic infants. Specifically, greater than or equal to $90 \%$ CPC (by the surgeon's intraoperative estimation) correlated with an $82 \%$ success rate of ETV/CPC in infants with hydrocephalus from various etiologies in a retrospective study by the Hydrocephalus Clinical Research Network. ${ }^{3}$ Additional studies have shown that the extent of CPC (along with other independent variables including age and hydrocephalus etiology) impacts the ETV/CPC success rates in Ugandan children with hydrocephalus. ${ }^{14,15}$ Therefore, radiographic markers that reflect the extent or completeness of CPC may help predict the success of ETV/CPC in hydrocephalic infants. The time course of CP disappearance on FS-MRI following CPC could not be evaluated in this study because of the retrospective nature of the data analysis and the variable timing of the imaging performed after surgery. Of note, 3 study patients underwent early followup imaging (11 days, 13 days, and 17 days after ETV/CPC), demonstrating $\mathrm{CP}$ persistence with subsequent imaging at later time points (12.1 weeks, 57.0 weeks, and 50.3 weeks, respectively) and showing $\mathrm{CP}$ disappearance without intervening surgery. Future prospective studies with imaging performed at specific time intervals may allow assessment of the time-dependent nature of $\mathrm{CP}$ disappearance following CPC.

\section{CSF Turbulence and CP Visualization in Combination}

Either or both radiographic markers appeared in $85 \%$ of "failed" FS-MRI scans of patients undergoing repeat treatment for hydrocephalus. This represented a statistically significant difference from the presence of either or both markers in only $28 \%$ of postoperative scans in patients who responded favorably to ETV/CPC ( $p=0.0007$; Table 4). Furthermore, the absence of both radiographic markers on postoperative imaging predicted clinical ETV/CPC success with moderate specificity (81\%; Table 4). With a higher sensitivity rating (91\%), CP persistence seemed to represent a stronger individual predictor of ETV/CPC failure than CSF turbulence visualization (sensitivity 80\%) in this retrospective study. However, the investigation of CSF turbulence in the context of treatment failure should not require a minimum time interval (occurring as early as 1.4 weeks postoperatively in 1 patient within this study) 
as may be required for $\mathrm{CP}$ evaluation following $\mathrm{CPC}$. Because it can be appreciated on postoperative imaging without the need for a minimum time interval for evaluation, radiographic CSF turbulence may reflect neuroendoscopic treatment failure on a time-independent, per-scan basis. In contrast, CP persistence (despite CPC) may reflect ETV/ CPC failure over time and ultimately require shunt insertion.

\section{Study Limitations}

Despite the potential applicability of these 2 radiographic markers, the weaknesses of this study should be recognized. The limited sample size (32 patients) and incorporation of 1 blinded observer represent quantitative shortcomings. This research effort represents a pilot study to explain the incorporation of a single, blinded observer. Future studies that include more study subjects and multiple observers with interobserver reliability estimates could corroborate and strengthen the current findings. The second stage of this research endeavor involves the analysis of the intra- and interobserver reliability of these radiographic markers, which is currently underway at the senior author's (C.J.R.) institution. Nevertheless, the method described in this study provided an estimation of an internal consistency rate exceeding $90 \%$, as described above. Additionally, the incorporation of a consistent imaging schedule following surgery in future prospective studies would provide more uniform data and may suggest the time course of CP disappearance following ETV/CPC. A minimum duration of clinical follow-up was not used here since this retrospective study highlighted the identification of the radiographic markers of ETV/CPC failure rather than prolonged success.

The uniform distinction of CSF turbulence and CP visualization represent qualitative challenges that can be mitigated by strict adherence to the requirements listed in Methods (Figs. 1, 2, and 4-6). The limited capabilities of FS-MRI necessitated the utilization of qualitative markers for CSF turbulence and CP visualization. Subjective interpretation of the absence of the $\mathrm{CP}$ glomus or the presence of only scant residual glomus within the ventricular atria qualified as surrogate markers for CP absence. While FSMRI represents a useful imaging modality in multiple age groups of patients who require radiographic evaluation following CSF diversion, these imaging sequences lack adequate resolution and anatomical definitions for consistently depicting the $\mathrm{CP}$ within the lateral ventricular temporal horns or bodies. Therefore, we selected the radiographic visualization of the $\mathrm{CP}$ glomus as the most reliable and consistent marker for determining adequate or inadequate CPC. This additionally reflects the intraoperative focus on thorough $\mathrm{CP}$ glomus cauterization during $\mathrm{CPC}$ at the senior author's (C.J.R.) institution. The use of the CP glomus as a surrogate marker of $\mathrm{CP}$ for determining the adequacy of CPC represents a study limitation imposed by the imaging modality (FS-MRI) that is most frequently used for clinical purposes in the infantile hydrocephalic population at the senior author's institution. However, head CT would likely result in similar imaging limitations, while standard MRI carries nontrivial risks and requirements for sedation and/or anesthesia in this young population.
Importantly, the modest sensitivity and specificity levels for the visualization of CSF turbulence and CP prohibit excessive or isolated reliance on these radiographic markers. These radiographic markers could be used in conjunction with other imaging modalities, including cardiac-gated MRI or CSF-flow studies, to help assess hydrocephalic infants following treatment as adjuncts to clinical evaluation.

\section{Conclusions}

This study presented 2 newly applied radiographic markers that are useful for the evaluation of hydrocephalic neonates and infants following ETV/CPC or under consideration for secondary shunting. The radiographic appearance of CSF turbulence may suggest the presence of pathological CSF flow dynamics and signal ETV/CPC failure, while $\mathrm{CP}$ persistence may predict the ultimate necessity for shunt insertion despite neuroendoscopic treatment. Radiographic evidence of adequate CPC, with the absence of CP on postoperative FS-MRI scans, suggested a higher likelihood of ETV/CPC success and shunt independence. The presence of either or both radiographic markers reflected ETV/CPC failure in a statistically significant fashion, but with only moderate sensitivity and specificity. Further prospective studies are warranted to confirm the predictive and clinical utility of these radiographic markers-CSF turbulence and CP visualization-in hydrocephalic neonates and infants undergoing ETV/CPC. Furthermore, future studies incorporating formal inter- and intraobserver reliability estimates may help corroborate the utility of these radiographic markers as adjuncts to clinical evaluation.

\section{References}

1. Di Rocco F, Grevent D, Drake JM, Boddaert N, Puget S, Roujeau T, et al: Changes in intracranial CSF distribution after ETV. Childs Nerv Syst 28:997-1002, 2012

2. Kulkarni AV, Drake JM, Armstrong DC, Dirks PB: Measurement of ventricular size: reliability of the frontal and occipital horn ratio compared to subjective assessment. Pediatr Neurosurg 31:65-70, 1999

3. Kulkarni AV, Riva-Cambrin J, Browd SR, Drake JM, Holubkov R, Kestle JR, et al: Endoscopic third ventriculostomy and choroid plexus cauterization in infants with hydrocephalus: a retrospective Hydrocephalus Clinical Research Network study. J Neurosurg Pediatr 14:224-229, 2014

4. Malko JA, Hoffman JC Jr, McClees EC, Davis PC, Braun IF: A phantom study of intracranial CSF signal loss due to pulsatile motion. AJNR Am J Neuroradiol 9:83-89, 1988

5. O'Hayon BB, Drake JM, Ossip MG, Tuli S, Clarke M: Frontal and occipital horn ratio: A linear estimate of ventricular size for multiple imaging modalities in pediatric hydrocephalus. Pediatr Neurosurg 29:245-249, 1998

6. Pindrik J, Jallo GI, Ahn ES: Changes in third ventricular size in pediatric patients undergoing endoscopic third ventriculostomy. Childs Nerv Syst 29:2027-2034, 2013

7. Scrivani PV, Freer SR, Dewey CW, Cerda-Gonzalez S: Cerebrospinal fluid signal-void sign in dogs. Vet Radiol Ultrasound 50:269-275, 2009

8. Sherman JL, Citrin CM: Magnetic resonance demonstration of normal CSF flow. AJNR Am J Neuroradiol 7:3-6, 1986

9. Sherman JL, Citrin CM, Bowen BJ, Gangarosa RE: MR demonstration of altered cerebrospinal fluid flow by obstructive lesions. AJNR Am J Neuroradiol 7:571-579, 1986 
10. Sherman JL, Citrin CM, Gangarosa RE, Bowen BJ: The MR appearance of CSF flow in patients with ventriculomegaly. AJR Am J Roentgenol 148:193-199, 1987

11. St George E, Natarajan K, Sgouros S: Changes in ventricular volume in hydrocephalic children following successful endoscopic third ventriculostomy. Childs Nerv Syst 20:834-838, 2004

12. Stone SS, Warf BC: Combined endoscopic third ventriculostomy and choroid plexus cauterization as primary treatment for infant hydrocephalus: a prospective North American series. J Neurosurg Pediatr 14:439-446, 2014

13. Warf BC: Congenital idiopathic hydrocephalus of infancy: the results of treatment by endoscopic third ventriculostomy with or without choroid plexus cauterization and suggestions for how it works. Childs Nerv Syst 29:935-940, 2013

14. Warf BC: The impact of combined endoscopic third ventriculostomy and choroid plexus cauterization on the management of pediatric hydrocephalus in developing countries. World Neurosurg 79:S23.e13-S23.e25, 2013

15. Warf BC, Mugamba J, Kulkarni AV: Endoscopic third ventriculostomy in the treatment of childhood hydrocephalus in Uganda: report of a scoring system that predicts success. J Neurosurg Pediatr 5:143-148, 2010

\section{Disclosures}

The authors report no conflict of interest concerning the materi- als or methods used in this study or the findings specified in this paper.

\section{Author Contributions}

Conception and design: Rozzelle, Pindrik, Rocque, Johnston. Acquisition of data: Rozzelle, Pindrik, Arynchyna. Analysis and interpretation of data: all authors. Drafting the article: Pindrik. Critically revising the article: all authors. Reviewed submitted version of manuscript: all authors. Statistical analysis: Rozzelle, Pindrik, Rocque. Administrative/technical/material support: Rozzelle. Study supervision: Rozzelle.

\section{Supplemental Information}

\section{Previous Presentations}

Portions of this work were presented in an oral format at the 43rd Annual Meeting of the American Association of Neurological Surgeons/Congress of Neurological Surgeons section on pediatric neurological surgery at Amelia Island, Florida, in December 2014.

\section{Correspondence}

Curtis J. Rozzelle, Division of Pediatric Neurosurgery, Department of Neurosurgery, Children's of Alabama and University of Alabama at Birmingham, 1600 7th Ave. South, Lowder 400, Birmingham, AL 35233. email: curtis.rozzelle@ childrensal.org. 